\title{
Opposite Effects of $\Delta$-9-Tetrahydrocannabinol and Cannabidiol on Human Brain Function and Psychopathology
}

\author{
Sagnik Bhattacharyya', Paul D Morrison², Paolo Fusar-Poli', ${ }^{1,3}$, Rocio Martin-Santos ${ }^{1,4}$, Stefan Borgwardt ${ }^{1,5}$, \\ Toby Winton-Brown', Chiara Nosarti ${ }^{6}$, Colin M O' Carroll', Marc Seal ${ }^{8}$, Paul Allen', Mitul A Mehta', \\ James M Stone', Nigel Tunstall' ${ }^{2}$, Vincent Giampietro' ${ }^{10}$, Shitij Kapur' ', Robin M Murray', \\ Antonio W Zuardi ${ }^{12,13}$, José A Crippa ${ }^{12,13}$, Zerrin Atakan' and Philip K McGuire' \\ 'Section of Neuroimaging, Division of Psychological Medicine \& Psychiatry, Institute of Psychiatry, King's College London, London, UK; ${ }^{2}$ Division of \\ Psychological Medicine \& Psychiatry, Institute of Psychiatry, King's College London, London, UK; ${ }^{3}$ Section of Psychiatry, Department of Health \\ Sciences, University of Pavia, Pavia, Italy; ${ }^{4}$ Department of Psychiatry, Institut of Neurosciences, Hospital Clínic, Barcelona, Spain; ${ }^{5}$ Psychiatric \\ Outpatient Department, University Hospital Basel, Basel, Switzerland; ${ }^{6}$ Cognition Schizophrenia and Imaging (CSI) Laboratory, Division of \\ Psychological Medicine \& Psychiatry, Institute of Psychiatry, King's College London, London, UK; ${ }^{7}$ Columbia University, Department of \\ Neuroscience, New York, NY, USA; ${ }^{8}$ Melbourne Neuropsychiatry Centre, The University of Melbourne, National Neuroscience Facility, Carlton \\ South, VIC, Australia; ${ }^{9}$ Centre for Neuroimaging Sciences, Institute of Psychiatry, KCL, London, UK; ${ }^{10}$ Department of Biostatistics, Institute of \\ Psychiatry, King's College London, London, UK; " Section on Schizophrenia, Imaging and Therapeutics, Division of Psychological Medicine \& \\ Psychiatry, Institute of Psychiatry, King's College London, London, UK; ${ }^{12}$ Department of Neuroscience and Behavior, Faculty of Medicine of \\ Ribeirão Preto, University of São Paulo, São Paulo, Brazil; ${ }^{3}$ INCT Translational Medicine, Ribeirão Preto, Brazil
}

$\Delta$-9-tetrahydrocannabinol ( $\Delta$-9-THC) and Cannabidiol (CBD), the two main ingredients of the Cannabis sativa plant have distinct symptomatic and behavioral effects. We used functional magnetic resonance imaging ( $\mathrm{fMRI}$ ) in healthy volunteers to examine whether $\Delta$-9-THC and CBD had opposite effects on regional brain function. We then assessed whether pretreatment with CBD can prevent the acute psychotic symptoms induced by $\Delta-9-\mathrm{THC}$. Fifteen healthy men with minimal earlier exposure to cannabis were scanned while performing a verbal memory task, a response inhibition task, a sensory processing task, and when viewing fearful faces. Subjects were scanned on three occasions, each preceded by oral administration of $\Delta-9-T H C, C B D$, or placebo. BOLD responses were measured using fMRI. In a second experiment, six healthy volunteers were administered $\Delta-9-\mathrm{THC}$ intravenously on two occasions, after placebo or CBD pretreatment to examine whether CBD could block the psychotic symptoms induced by $\Delta$-9-THC. $\Delta$-9-THC and CBD had opposite effects on activation relative to placebo in the striatum during verbal recall, in the hippocampus during the response inhibition task, in the amygdala when subjects viewed fearful faces, in the superior temporal cortex when subjects listened to speech, and in the occipital cortex during visual processing. In the second experiment, pretreatment with CBD prevented the acute induction of psychotic symptoms by $\Delta$-9-tetrahydrocannabinol. $\Delta$-9-THC and CBD can have opposite effects on regional brain function, which may underlie their different symptomatic and behavioral effects, and CBD's ability to block the psychotogenic effects of $\Delta$-9-THC.

Neuropsychopharmacology (2010) 35, 764-774; doi:10.1038/npp.2009. 184; published online I8 November 2009

Keywords: $\Delta$-9-tetrahydrocannabinol; Cannabidiol; psychosis; anxiety; fMRI

\section{INTRODUCTION}

In healthy individuals, $\Delta$-9-tetrahydrocannabinol ( $\Delta-9$ THC), the main psychoactive ingredient of the Cannabis sativa plant, can induce psychotic symptoms and anxiety, and can impair memory (D'Souza et al, 2004) and psychomotor control (McDonald et al, 2003; Ramaekers et al,

*Correspondence: Dr S Bhattacharyya, Kings College London, Section of Neuroimaging, Box P067, Institute of Psychiatry, De Crespigny Park, London SE5 8AF, UK, Tel: + 44207848 0955,

Fax: + 44207848 0976, E-mail: s.bhattacharyya@iop.kcl.ac.uk Received 20 May 2009; revised 30 September 2009; accepted 30 September 2009
2006). In patients with schizophrenia, $\Delta$-9-THC may exacerbate existing psychotic symptoms, anxiety and memory impairments (D'Souza et al, 2005), and $\Delta$-9-THC is thought to be the ingredient responsible for the increased risk of developing schizophrenia following regular cannabis use (Moore et al, 2007). In contrast, Cannabidiol (CBD), the other major psychoactive constituent of C. sativa, has anxiolytic (Crippa et al, 2004) and possibly antipsychotic properties (Zuardi et al, 2006; Morgan and Curran, 2008; Zuardi, 2008), does not impair memory or other cognitive functions (Fadda et al, 2004; Ilan et al, 2005). Although CBD has been shown to have neuroprotective effects (Hampson et al, 1998; Mechoulam et al, 2002; Lastres-Becker et al, 
2005), $\Delta$-9-THC may have neurotoxic as well as neuroprotective effects (Sarne and Mechoulam, 2005). Moreover, when co-administered with $\Delta-9$-THC, CBD may be able to reduce some of the symptomatic effects of $\Delta$-9-THC like anxiety and paranoia (Karniol et al, 1974; Dalton et al, 1976; Zuardi et al, 1982). CBD may thus have therapeutic potential as a treatment for cannabis-induced psychopathology, and as an anxiolytic and an antipsychotic (Zuardi, 2008). However, none of the earlier studies (Karniol et al, 1974; Dalton et al, 1976; Zuardi et al, 1982) had used standardized rating scales to formally assess psychotic symptoms.

The neural basis for these distinct effects of $\Delta-9$-THC and CBD on psychiatric symptoms and cognitive function is unclear. Recent data from experimental animals and in vitro studies suggest that $\Delta-9$-THC and CBD may have opposing effects on brain cannabinoid (CB1) receptors (Pertwee, 2008). Although the effects of $\Delta-9$-THC are thought to be mediated by a partial agonism at the central CB1 receptors (Pertwee, 2008), the precise molecular mechanism of action of CBD is unclear and may involve a wide variety of mechanisms (Mechoulam et al, 2007). In the absence of this information, examination of the downstream effects of CBD in the brain in terms of neural activation and behavior provides another mode of unravelling its effects. To date, functional neuroimaging studies of $\Delta-9$-THC and CBD in man have examined the effects of each compound separately, but have not compared them with each other directly (Borgwardt et al, 2008; Phan et al, 2008; Bhattacharyya et al, 2009; Fusar-Poli et al, 2009). In this study, we sought to address this issue by using functional magnetic resonance imaging (fMRI) to contrast the acute effects of $\Delta-9$-THC and CBD on regional brain function and the mental state in the same healthy volunteers. We then examined their interaction at the behavioral level, by assessing the extent to which pretreatment with CBD could prevent the acute symptomatic effects of subsequently administered $\Delta$-9-THC. The first hypothesis tested was that $\Delta$-9-THC and CBD would have opposite effects on regional brain activation during a set of four tasks that engaged cognitive processes known to be affected by cannabis use: verbal memory, response inhibition, sensory processing, and emotional processing (Hall and Solowij, 1998).
Our second hypothesis was that CBD pretreatment would diminish the effects of $\Delta$-9-THC-induced psychotic symptoms.

\section{MATERIALS AND METHODS}

The protocols used in this experimental study were approved by the Joint South London and Maudsley/Institute of Psychiatry NHS Research Ethics Committee. All participants provided written informed consent, which was obtained after they were explained the study design and the risk of transient psychotic symptoms and anxiety associated with the administration of $\Delta-9$-THC.

\section{fMRI Experiments}

Participants were studied using a 1.5-T GE scanner on three occasions, at 1-month intervals, following administration of either $10 \mathrm{mg}$ of $\Delta-9$-THC, $600 \mathrm{mg}$ of CBD or placebo, in a double-blind, repeated measures, within-subject design. Order of drug administration was pseudorandomizsed across subjects, so that an equal number of subjects received any of the drugs during the first, second, or third session. On each occasion, participants performed four cognitive tasks inside the scanner, which were always presented in the same order: (1) verbal memory, (2) response inhibition, (3) viewing fearful faces, and (4) visual and auditory stimulation task.

Experimental procedure. Fifteen healthy right-handed English-speaking men (National Adult Reading Test IQ-98.7) (Nelson, 1982), who had been exposed to cannabis $<15$ times in their life but not in the past month, participated (Table 1). None of the subjects had any personal or family history of psychiatric illness and alcohol or other drug abuse or dependence. They had minimal exposure to other illicit drugs in the form of experimentation (Table 1). Experimentation typically involved use of the drug on $<3$ occasions lifetime. All subjects were requested to abstain from use of recreational drugs for the duration of the study, alcohol intake for $24 \mathrm{~h}$ and caffeine intake for $12 \mathrm{~h}$ before the study and also to avoid smoking tobacco

Table I Subject Characteristics

\begin{tabular}{|c|c|c|}
\hline & fMRI experiments $(n=15)$ & Behavioral experiment $(n=6)$ \\
\hline Gender & Males $=15$ & Females $=3 ;$ males $=3$ \\
\hline $\begin{array}{l}\text { Mean age (SD) } \\
\text { (years) }\end{array}$ & $26.7(5.7)$ & $25.6(8.2)$ \\
\hline Age range & $20-42$ & $21-42$ \\
\hline $\begin{array}{l}\text { Cannabis use } \\
\text { (lifetime) }\end{array}$ & $<5$ times: 9 subjects; $5-14$ times: 6 subjects & Mean use: 150 times \\
\hline $\begin{array}{l}\text { Other illicit drug } \\
\text { use (lifetime) }\end{array}$ & $\begin{array}{l}\text { Amphetamines, } 3 \text { subjects }{ }^{\mathrm{a}} \text {; LSD/Psilocybin, } 3 \text { subjects; }{ }^{\mathrm{a}, \mathrm{b}} \text { MDMA, } 5 \\
\text { subjects; }{ }^{\mathrm{b}} \text { Cocaine, I subject }\end{array}$ & $\begin{array}{l}\text { Cocaine only - I subject; Cocaine, LSD/Psilocybin, ketamine, MDMA, } \\
\text { I subject }\end{array}$ \\
\hline $\begin{array}{l}\text { Current tobacco } \\
\text { smokers }\end{array}$ & $\begin{array}{l}7 \text { subjects; mean number of cigarettes smoked/day, } 2.92 \text { (SD, 4.9) } \\
\text { (range, } 0-15 / \text { day); } 2 \text { subjects smoked }>10 \text { cigarettes/day }\end{array}$ & $\begin{array}{l}3 \text { subjects; mean number of cigarettes smoked/day, } 5.0 \text { (SD, 6.3) } \\
\text { (range, } 0-15 / \text { day); I subject smoked }>10 \text { cigarettes/day }\end{array}$ \\
\hline
\end{tabular}

a subject had experimented with both amphetamines and LSD/Psilocybin.

${ }^{b}$ I subject had experimented with both LSD/Psilocybin and MDMA. 
(Table 1) on the morning of the study. Two hours before each session, subjects had a light standardized breakfast. One hour before scanning, they were given a gelatin capsule containing either $10 \mathrm{mg}$ of $\Delta-9$-THC, $600 \mathrm{mg}$ of CBD (THCPharm, Frankfurt, Germany) or flour (placebo). All subjects had a negative urinary drug screen before all sessions. Illicit substance use was assessed using the Structured Clinical Interview and Addiction Severity Index (McLellan et al, 1992). Psychopathology was assessed using the Visual Analogue Mood Scale (VAMS) (Folstein and Luria, 1973), the Spielberger State Trait Anxiety Inventory (STAI) (Spielberger, 1983), and the Positive and Negative Syndrome Scale (PANSS) (Kay et al, 1987). Heart rate and blood pressure were monitored through a digital recorder and an automated arm cuff. Blood samples were taken from an indwelling intravenous line in the nondominant arm. Whole-blood drug levels were measured by Tricho Tech (Cardiff, UK). Skin conductance response (SCR) was recorded through electrodes on the fingers of the nondominant hand while performing the 'viewing fearful faces' task (please see below), with fluctuations defined as an increase of $>0.01$ ís with respect to each pretarget stimulus baseline and occurring $0.5-3 \mathrm{~s}$ after the target face stimulus. Psychopathological ratings and venous blood samples were obtained immediately before, and at 1,2 , and $3 \mathrm{~h}$ after drug administration. Scanning was performed between 1 and $2 \mathrm{~h}$ after drug administration.

\section{Cognitive tasks.}

Verbal memory task: This task has been described in detail earlier (Bhattacharyya et al, 2009) and hence described here briefly (Figure 1a). During an encoding condition, subjects were shown pairs of words and indicated whether they went well together by saying 'Yes' or 'No' (to encourage encoding). During a recall condition, one word from each pair was presented next to a question mark and subjects had to articulate the word it had previously been paired with, saying 'pass' if they could not recall it. In a baseline condition, subjects were shown pairs of words printed with same or different fonts taken from a different list of words. Stimuli were presented in $40 \mathrm{~s}$ blocks comprising eight stimulus pairs, with the conditions repeated in the same sequence four times.
Viewing fearful faces: This task has been described in detail earlier (Fusar-Poli et al, 2009) and hence described here briefly (Figure 1b). Subjects were presented with a series of 10 different facial identities, each expressing either a $50 \%$ (mildly fearful) or $100 \%$ (prototypically fearful) intensity of fear, or a neutral expression. There were thus 30 different facial stimuli in total. Each stimulus was presented for $2 \mathrm{~s}$ on two occasions with the order of identities and expression pseudorandomized. The mean interstimulus interval was $5.9 \mathrm{~s}(3-8 \mathrm{~s})$ during which time subjects viewed a fixation cross. Subjects were told to indicate the gender of each face by pressing one of two buttons.

Response inhibition task (Go No-Go): This task has also been described in detail earlier (Borgwardt et al, 2008) and hence described here briefly (Figure 1c). Subjects were presented with a series of arrows, visible for $500 \mathrm{~ms}$ with a mean interstimulus interval of $1.8 \mathrm{~s}$. When arrows pointed left or right (Go trials), subjects were required to press a left or right response button accordingly. On $12 \%$ of trials (No-Go trials), arrows pointing upwards were presented and subjects were required to not press any button. On another $12 \%$ of trials, arrows pointing obliquely left or right were presented, which subjects were told to respond to as for a 'Go' stimulus. These 'Oddball' stimuli were used to control for novelty effects associated with the low frequency and different orientation of No-Go relative to Go stimuli, and were used as the contrast condition for the No-Go trials. A total of $184 \mathrm{Go}, 24 \mathrm{No}-\mathrm{Go}$, and 24 Oddball stimuli were presented in random order in an event-related design.

During all of the above tasks, the speed and accuracy of behavioral responses was recorded on-line.

Visual and auditory stimulation: During each of the $16 \mathrm{~s}$ blocks of a visual stimulation condition (Figure 1d), subjects viewed a radial chequerboard (flicker rates randomly varied at 2,4 , and $8 \mathrm{~Hz}$ ). During each of the $24 \mathrm{~s}$ blocks of an auditory stimulation condition, subjects listened to neutral words (read at randomly varied rates of 30 , 60, and 90 words per minute) presented through headphones. The visual stimulation blocks were presented four times and the auditory stimulation blocks were presented three times.

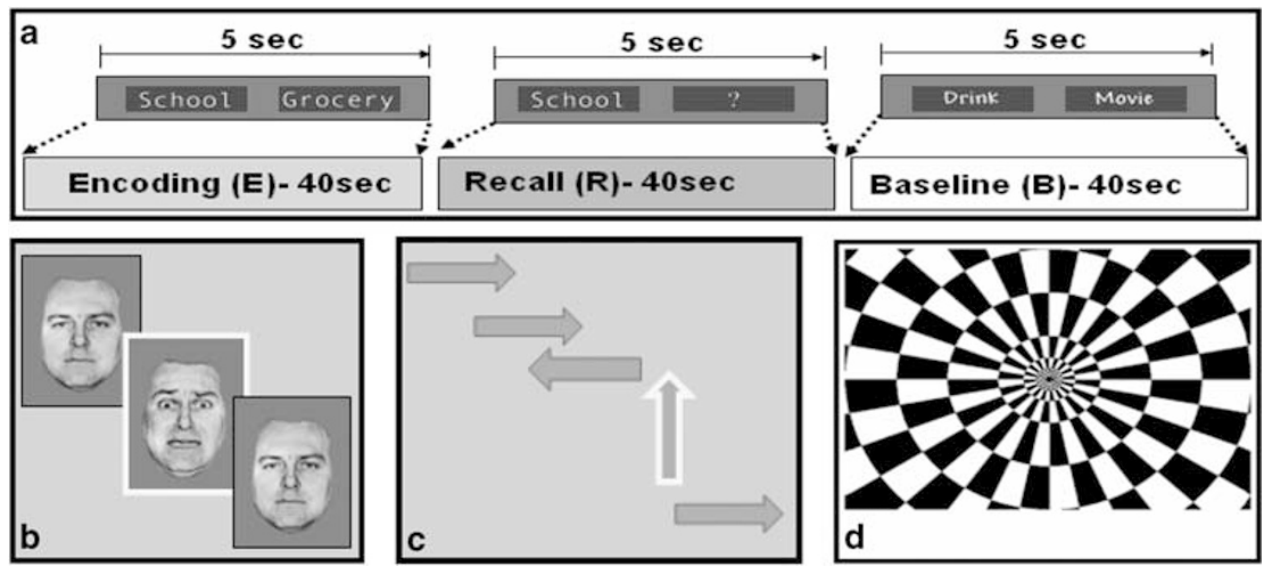

Figure I Cognitive tasks performed during the fMRI experiments: (a) verbal memory, (b) viewing fearful faces, (c) response inhibition, and (d) visual processing. 
Image acquisition: Images were acquired on a 1.5-T (GE) system. T2*-weighted images were acquired with TE $40 \mathrm{~ms}$, flip angle $90^{\circ}$ in 16 axial planes ( $7 \mathrm{~mm}$ thick), parallel to the AC-PC line. The TR during the Go No-Go, fearful faces, and visual and auditory stimulation paradigms was $2 \mathrm{~s}$. During the verbal paired associates task, a compressed acquisition with TR $5 \mathrm{~s}$ and $3.5 \mathrm{~s}$ of silence was used. A high-resolution inversion recovery image dataset was also acquired to facilitate anatomical localization of activation.

\section{Behavioral Experiment}

Although the fMRI study was designed to address the question whether $\Delta-9-$ THC and CBD have opposite effects at the neurophysiological level, it could not adequately address the issue of whether they have opposite effects at the behavioral level. Hence, we conducted the behavioral experiment to provide clear evidence whether pretreatment with CBD can prevent $\Delta-9$-THC from provoking psychotic symptoms in healthy subjects.

Six healthy English-speaking volunteers participated in two experimental sessions at least 2 weeks apart. They had a mean lifetime cannabis use history of 150 times and had minimal exposure to other illicit drugs (Table 1). All subjects had a negative urinary drug screen before the sessions. Using a repeated measures, pseudorandomized, double-blind, within-subject design, 'Pretreatments,' CBD $(5 \mathrm{mg})$, or placebo were administered intravenously (IV) over $5 \mathrm{~min}$ immediately before IV $\Delta$-9-THC $(1.25 \mathrm{mg})$, which was also administered over $5 \mathrm{~min}$. Positive psychotic symptoms were assessed at baseline and at 30 and $90 \mathrm{~min}$ post- $\Delta-9-\mathrm{THC}$, by an independent psychiatrist using the PANSS rating scale.

\section{Data Analysis}

Data from the fMRI tasks were analyzed using XBAMv3 (http://wwwbrainmap.it/). Images were realigned and smoothed with an 8-mm Gaussian filter. Individual activation maps were created using 2 ã-variate functions to model the BOLD response. Following a least squares fitting of this model, the sum of squares ratio (SSQ) was estimated at each voxel, followed by permutation testing to determine significantly activated voxels specific to each condition (Bullmore et al, 2001). SSQ ratio maps for each individual were transformed into standard stereotactic space (Talairach and Tournoux, 1988) and group activation maps computed for each drug by determining the median SSQ ratio at each voxel. These maps were compared using nonparametric-repeated measures ANCOVA, with a voxelwise threshold of $p=0.05$ and the cluster-wise threshold set such that the total number of false positive clusters per brain volume was $<1$ : the $p$-value at which the latter occurred is quoted. The principal advantages of cluster-level testing are that it confers greater sensitivity by incorporating information from more than one voxel in the test statistic and also substantially reduces the search volume or number of tests required for a whole-brain analysis, thereby mitigating the multiple comparisons problem.

For each drug condition ( $\Delta-9-\mathrm{THC}, \mathrm{CBD}$, and placebo), we first contrasted the active task condition against the baseline condition. For the retrieval condition of the verbal memory task, this involved contrasting all the recall blocks independent of repetition against the baseline blocks for each drug treatment $(\Delta-9-\mathrm{THC}, \mathrm{CBD}$, and placebo), to control for activation related to processing visually presented words. For the emotional processing condition (viewing fearful faces task), this involved contrasting the $100 \%$ fearful faces against neutral faces for each drug treatment, to control for activation related to processing faces independent of their emotional expression. For the event-related analysis of the response inhibition condition (Go No-Go task), this involved first contrasting the 'No-Go' and 'Oddball' trials against the 'Go' trials for each drug treatment, to control for activation related to the processing of visually presented arrows on a screen. Brain activation during the successfully performed 'Oddball' trials, which controlled for novelty effects, was then subtracted from brain activation during the successful 'No-Go' trials ('NoGo' minus 'Oddball') for each drug condition, to derive brain activation related to response inhibition. For the sensory processing condition (visual and auditory stimulation task), this involved contrasting the task condition (auditory or visual stimuli) independent of the sensory load against the fixation cross, for each drug treatment. Finally, in keeping with our hypothesis that $\Delta-9$-THC and CBD would have opposite effects on regional brain activation, we selectively identified areas where the effects of $\Delta-9$-THC and CBD were in the opposite direction relative to the placebo condition for each task condition (verbal memory retrieval, emotional processing, response inhibition, sensory processing) using a nonparametric-repeated measures ANOVA.

Measures of task performance, symptom ratings, physiological data, and drug levels for the fMRI experiment were analyzed using repeated measures ANOVAs used to compare drug conditions. When significant differences were found, the Tukey's test for pairwise comparisons was applied. However, the small sample size for the behavioral experiment precluded a parametric approach for which a nonparametric statistical test (Kolmogorov-Smirnov one sample test) was used. The effects of between-drug differences in symptom levels on activation were examined by correlating measures of activation with the change in the rating from baseline to the mean of those at 1 and $2 \mathrm{~h}$. Cook's distance test was used to assess the robustness of correlations.

\section{RESULTS}

\section{fMRI Experiments}

Behavioral effects of $\triangle-9-T H C$ and CBD. For the sake of completeness, we have reported the symptomatic and behavioral effects from the fMRI experiments briefly here. They have already been reported in detail earlier (Borgwardt et al, 2008; Bhattacharyya et al, 2009; Fusar-Poli et al, 2009). Oral administration of $\Delta-9$-THC was associated with the acute induction of both psychotic symptoms $(p<0.01)$ as measured using PANSS positive symptoms subscale (Figure 2a) and anxiety $(p<0.01)$ measured using STAI trait subscale, (Figure $2 \mathrm{~b}$ ), whereas following CBD there was no change in psychotic symptoms, and a trend for a reduction in subjective anxiety $(p=0.06)$ measured using the VAMS tranquilization and calming subscale (Figure 2c). There was 

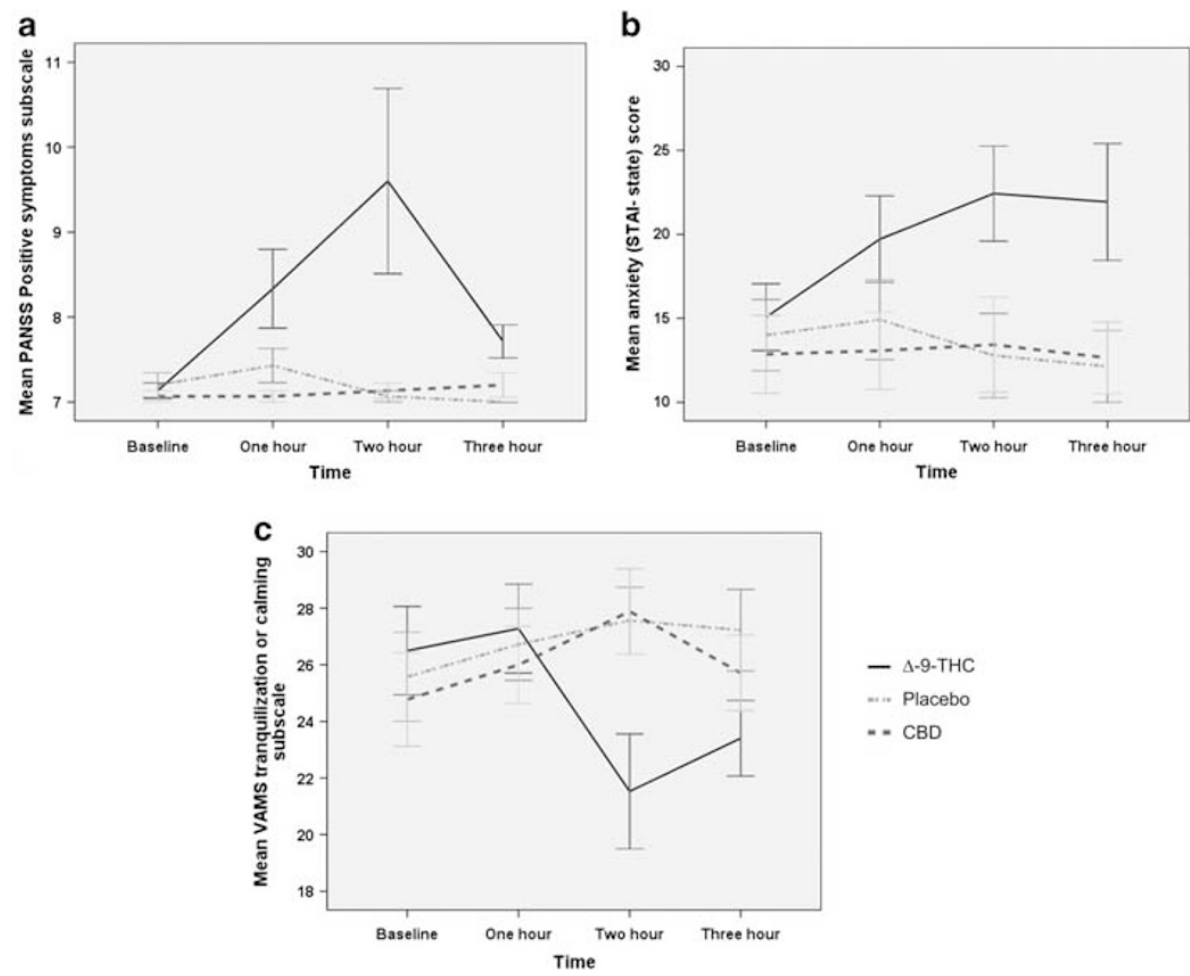

Figure 2 Plots showing changes in psychotic symptoms as indexed by PANSS positive symptoms subscale (a), anxiety as indexed by the State Trait Anxiety Inventory (STAI) state (b), VAMS tranquilization or calming subscale (c) following oral administration of $\Delta$-9-THC, CBD, or placebo during the fMRI experiments.

a nonsignificant trend for an increase in heart rate (beats/ min) with $\Delta$-9-THC $[+1.93( \pm 5.74)$ and $+8.79( \pm 16.31)$ at 1 and $2 \mathrm{~h}$ after baseline]. Blood levels of $\Delta-9$-THC were $3.9(\mathrm{SD}=7.3)$ and $5.1(5.6) \mathrm{ng} / \mathrm{ml}$ at 1 and $2 \mathrm{~h}$, respectively, whereas blood levels of CBD were $4.7(7.0) \mathrm{ng} / \mathrm{ml}$ after $1 \mathrm{~h}$ and $17(29.0) \mathrm{ng} / \mathrm{ml}$ after $2 \mathrm{~h}$. Neither drug had significant effects on behavioral performance of the verbal memory [Mean retrieval score \pm SD (number of words recalled): $\Delta$-9-THC-29.87 \pm 3.16 ; placebo-30.27 \pm 3.15 ; CBD-30.87 \pm 1.36], viewing fearful faces [accuracy of gender discrimination \pm SD (\%): $\Delta-9-$ THC-82.49 \pm 3.86 ; placebo-83.45 \pm 2.63 ; CBD-83.44 \pm 3.16 ] or the response inhibition [probability of inhibition \pm SD (\%): $\Delta$-9-THC-93.6 \pm 8.5 ; placebo-95.3 \pm 7.9 ; CBD-96.4 \pm 6.3 ] tasks $(p>0.1)$.

Opposite neurophysiological effects of $\triangle-9-T H C$ and CBD. During the verbal memory task, $\Delta-9$-THC and CBD had opposite effects on activation during the retrieval phase in the striatum, and the anterior cingulate/medial prefrontal and lateral prefrontal cortex (Figure $3 \mathrm{a}$ and b) (Table 2). The effect of $\Delta-9-$ THC in the striatum was inversely correlated with the severity of the psychotic symptoms (as indexed by the PANSS positive symptoms subscale) it concurrently induced: the more it attenuated striatal activation, the more severe were the psychotic symptoms ( $r=-0.574, p=0.013$; after leaving outliers identified by Cook's D reliability analysis: $r=-0.805, p<0.001)$. This relationship between brain activation and psychotic symptoms was not evident in any of the other brain areas where the opposite effects of $\Delta-9$-THC and CBD were identified during the verbal memory task.
While viewing fearful faces, $\Delta$-9-THC and CBD had opposite effects on activation in the left amygdala, fusiform, and lingual gyri, the lateral prefrontal cortex and the cerebellum (Figure $3 c$ and $d$ ) (Table 2). In the amygdala, $\Delta$-9-THC augmented activation in response to fearful faces, and this effect was directly correlated with the associated level of anxiety, as indexed by the STAI $(r=0.675, p=0.003)$; in contrast, $\mathrm{CBD}$ attenuated the amygdalar response and this effect was correlated $(r=0.551, p=0.017)$ with its trend level anxiolytic effect, as indexed by the VAMS tranquilization and calming subscale. $\Delta$-9-THC and CBD also had opposite effects on the number of SCR fluctuations while viewing intensely fearful faces. Although $\Delta-9$-THC increased the number of SCR fluctuations $(p<0.05)$, CBD resulted in a decrease $(p<0.05)$ in the number of SCR fluctuations relative to placebo. This effect of $\mathrm{CBD}$ on the number of SCR fluctuations was correlated $(r=0.524 ; p=0.049)$ with the attenuation of amygdala response it concurrently induced.

During the Go No-Go task, the two drugs had opposite effects in the parahippocampal gyrus bilaterally, the left insula and caudate. In these regions, $\Delta-9$-THC attenuated activation, whereas $\mathrm{CBD}$ augmented activation relative to placebo (Figure 4a and b; Table 2).

When subjects were listening to speech, $\Delta-9$-THC and CBD had opposite effects on activation in the lateral temporal cortex bilaterally (Figure $4 \mathrm{c}$ and $\mathrm{d}$; Table 2). When they were viewing a visual checkerboard, the drugs had opposite effects relative to placebo in the occiptal cortex, bilaterally (Figure $4 \mathrm{e}$ and f; Table 2). 

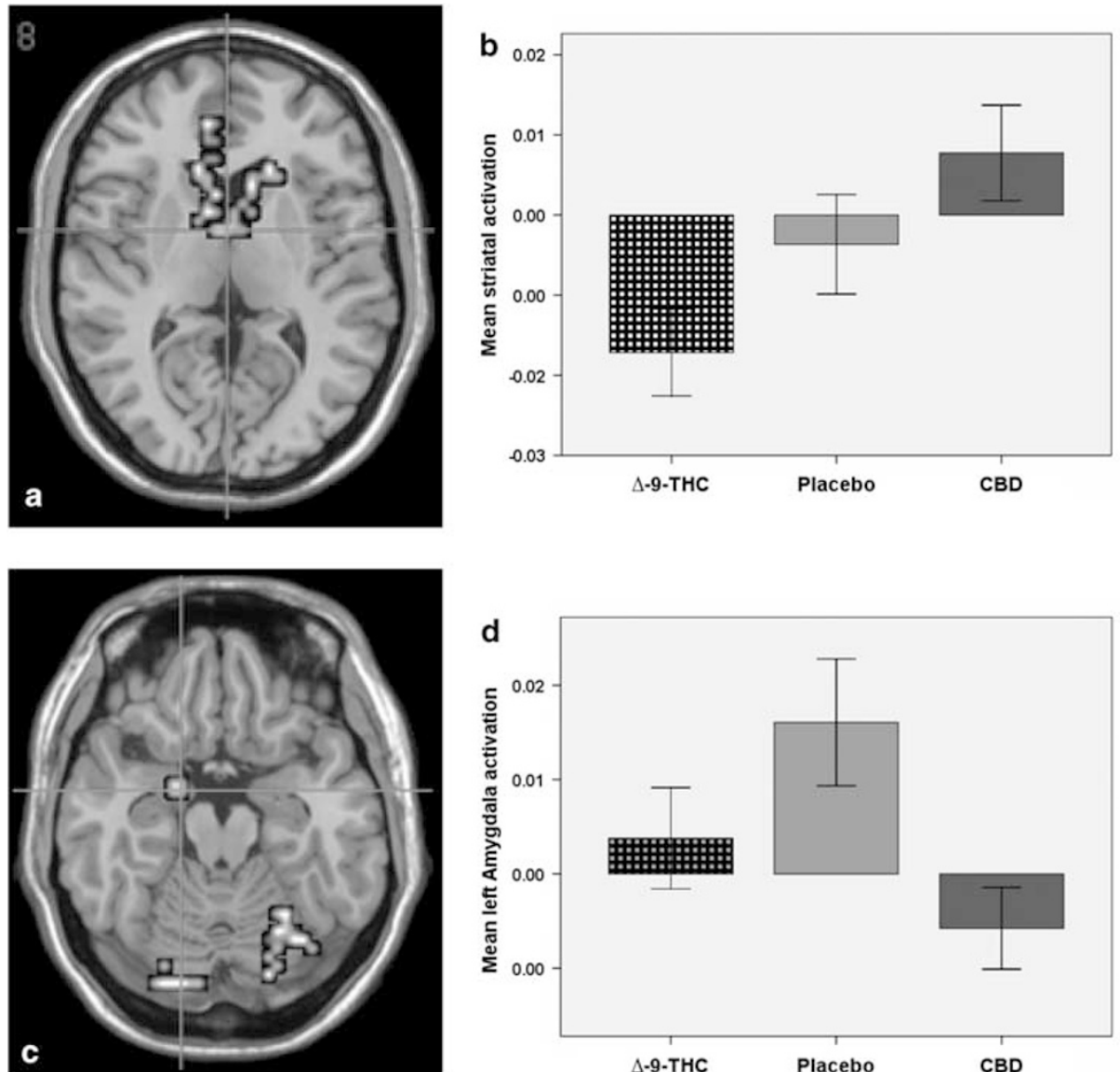

Figure 3 (a) Opposite effects of $\Delta-9-$ THC and CBD on prefrontal and striatal activation during word retrieval. The left side of the brain is shown on the left side of the images. (b) The bar graph (mean \pm SEM) shows that striatal activation ( $x$ axis; arbitrary units) in (a) was attenuated by $\Delta$-9-THC but augmented by CBD. (c) Opposite effects of $\Delta-9-T H C$ and CBD on amygdalar activation while subjects viewed fearful faces. The left side of the brain is shown on the left side of the images. (d) The bar graph (mean \pm SEM) shows that amygdalar activation ( $x$ axis; arbitrary units) in (c) was augmented by $\Delta$-9-THC and placebo but attenuated by CBD.

\section{Behavioral Experiment}

Blood levels of $\Delta-9$-THC as measured using area under the curve from time 0 to 120 min $\left(\mathrm{AUC}_{0-120}\right.$, mean $\left.\pm \mathrm{SD}\right)$ was $888.1 \pm 132.4 \mathrm{ng} \mathrm{min} / \mathrm{ml}$ under the placebo pretreatment condition and $1211.7 \pm 395.2 \mathrm{ng} \mathrm{min} / \mathrm{ml}$ under the CBD pretreatment conditions. These were not significantly $(p>0.05)$ different. Three of the six volunteers experienced psychotic symptoms when given $\Delta-9$-THC following placebo. In all three of these subjects, pretreatment with CBD diminished the emergence of psychotic symptoms measured $30 \mathrm{~min}$ after administration of $\Delta-9$-THC, reducing the mean PANSS positive score from $13(\mathrm{SD}=5.8)$ to $9(\mathrm{SD}=2.2)$ (Figure $5 \mathrm{a}$ and b). At $30 \mathrm{~min}$ after administration of $\Delta$-9-THC, psychotic symptoms (5-factor PANSS) induced by $\Delta$-9-THC were significantly lower under the CBD compared with the placebo pretreatment condition $(z=-2.3, p<0.05)$.

\section{DISCUSSION}

This study investigated whether, following oral administration, CBD would have an opposite neural effect to that of $\Delta$-9-THC during the performance of various cognitive tasks known to be modulated by cannabis. Further, it examined whether CBD would oppose the psychotic symptoms induced by $\Delta-9$-THC when co-administered IV to healthy individuals. A striking finding was that the net effects of orally administered $\Delta-9$-THC and CBD on activation relative to placebo were in the opposite direction in the context of a series of tasks that engaged a diversity of brain regions and cognitive processes. Thus, these opposite effects were evident in the striatum, anterior cingulate/medial prefrontal cortex, and lateral prefrontal cortex during verbal recall, the parahippocampal gyrus during response inhibition, the amygdala during the processing of fearful faces, and the temporal and occipital cortices during auditory and visual processing, respectively. This suggests that this difference in the effects of $\Delta-9$-THC and CBD is not specific to a particular brain system, and may be common to multiple regions and cognitive processes. At the same time, because the analysis selectively identified areas in which opposite effects were evident, it is entirely possible that there is a different relationship between the effects of the two compounds in other brain areas. This possibility could be tested in a separate study.

Opposite effects of $\Delta-9-\mathrm{THC}$ and CBD on activation in the anterior cingulate/medial prefrontal cortex and the lateral prefrontal cortex in the context of verbal recall is consistent 
Table 2 Talairach Coordinates of Peak Areas of Activation Where $\Delta$-9-THC and CBD had Opposite Effects During the Various Task Conditions

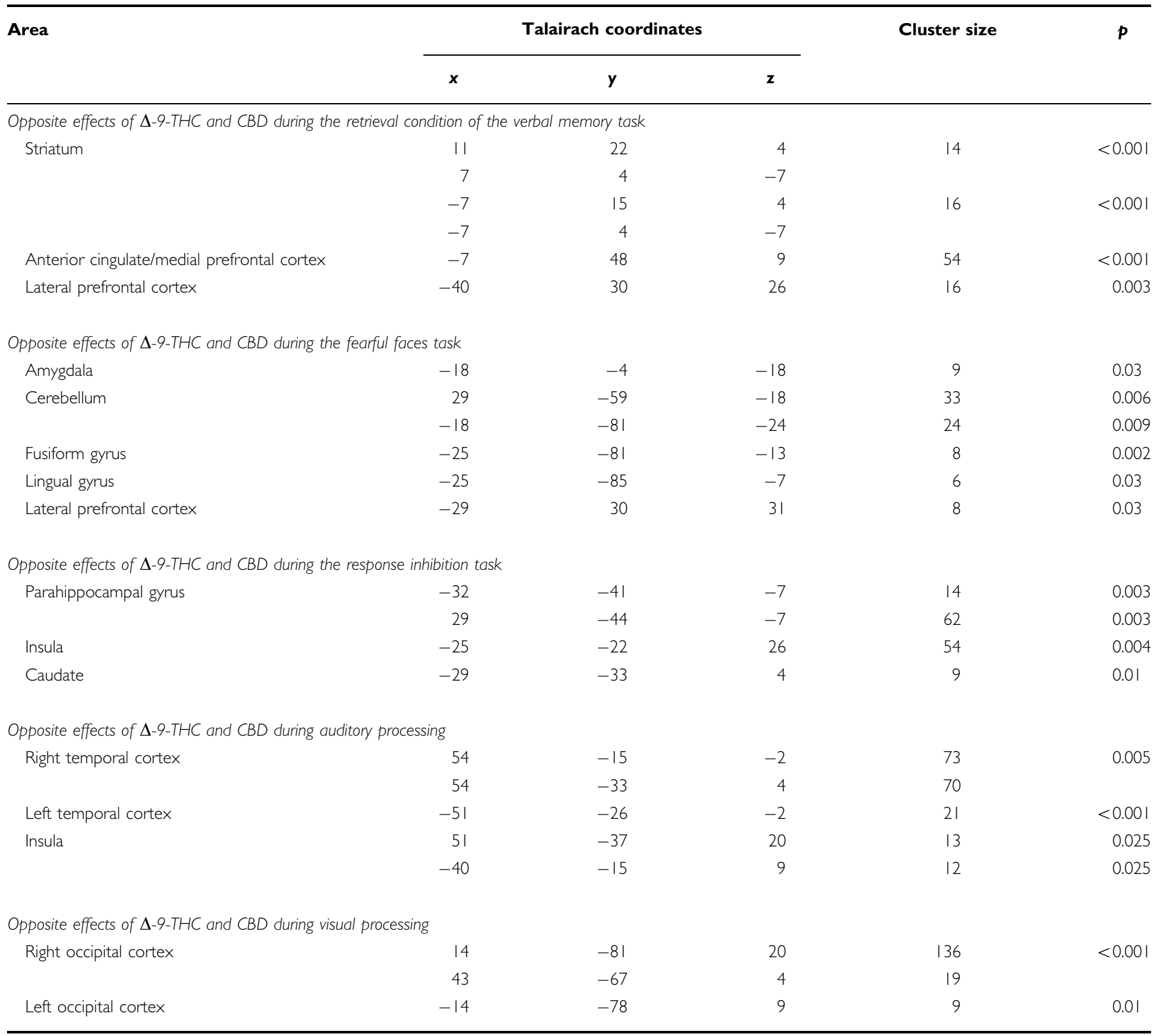

with the key role played by these regions in different aspects of retrieval from control processes necessary for the specification of retrieval to monitoring and verification (Simons et al, 2005; Fleck et al, 2006; Cabeza and St Jacques, 2007) and may reflect differential engagement of these processes under the drug conditions. Modulation of striatal activation during verbal recall may reflect increased ease of responding as subjects learned the responses to recall cues as they were repeatedly presented (Doeller et al, 2008). Attenuation of striatal activation by $\Delta-9$-THC may be interpreted in terms of a perturbation of the neural correlate of the increasing ease of recall responses, whereas the opposite effect of CBD may be interpreted as a facilitation of this process. Opposite effects of $\Delta-9-$ THC and CBD on amygdala activation during fear processing is consistent with the key role played by amygdala in the processing of fear (Morris et al, 1996; Breiter et al, 1996) as well as evidence that CBD (Crippa et al, 2004) and $\Delta$-9-THC (Phan et al, 2008) modulate amygdala activation in the context of anxiety. However, there is an apparent discrepancy between the results of this study with the study by Phan et al (2008) who showed the anxiolytic role of a smaller dose of $\Delta$-9-THC mediated through its effect on amygdala (Phan et al, 2008). But, they may be reconciled by the biphasic effects of $\Delta-9$-THC and other cannabinoids on anxiety with lower doses generally being anxiolytic and higher doses being anxiogenic (Viveros et al, 2005). Although the parahippocampal areas are not part of the response 

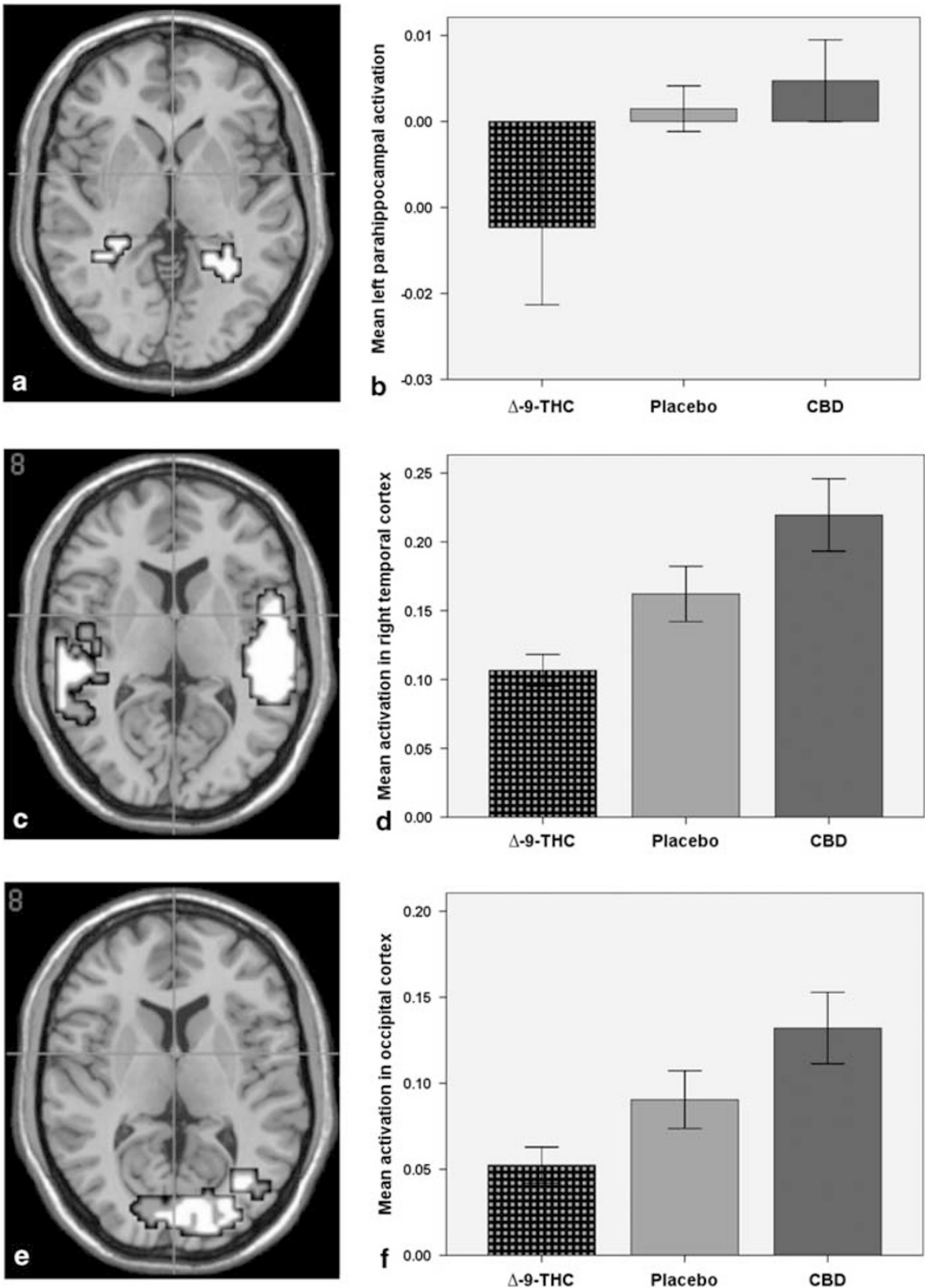

Figure 4 (a) Opposite effects of $\Delta-9-\mathrm{THC}$ and CBD on parahippocampal activation during a response inhibition task. The left side of the brain is shown on the left side of the images. (b) The bar graph (mean \pm SEM) shows that left parahippocampal activation ( $x$ axis; arbitrary units) in (a) was attenuated by $\Delta$ 9-THC but augmented by CBD. (c) Opposite effects of $\Delta-9-$ THC and CBD on temporal activation during auditory processing. The left side of the brain is shown on the left side of the images. (d) The bar graph (mean \pm SEM) shows that activation ( $x$ axis; arbitrary units) in the right temporal cortex in (c) was attenuated by $\Delta-9-$ THC relative to placebo but augmented by CBD. (e) Opposite effects of $\Delta-9-T H C$ and CBD on occipital activation during visual processing. The left side of the brain is shown on the left side of the images. ( $f$ ) The bar graph (mean \pm SEM) shows that activation ( $x$ axis; arbitrary units) in the right occipital cortex in (e) was attenuated by $\Delta-9-$ THC relative to placebo but augmented by CBD.

inhibition network (Rubia et al, 2001), opposite effects of $\Delta$-9-THC and CBD in the parahippocampal areas during the response inhibition task is consistent with the high density of $\mathrm{CB} 1$ receptors in these regions (Elphick and Egertova, 2001) and may partly reflect the effect of these drugs during the 'Oddball' condition. Finally, modulation of the lateral temporal and occipital cortices by these cannabinoids during an auditory and visual processing task, respectively, is also consistent with the role played by these regions in auditory (Price et al, 1996) and visual (Haxby et al, 1991) processing as well as evidence that cannabis has marked effect on sensory experiences (Tart, 1970) and modulates auditory processing related activity in the temporal cortex (O'Leary et al, 2002). The widely distributed brain regions where $\Delta$-9-THC and CBD had opposite effects is also consistent with the distribution of CB1 receptors in the brain (Elphick and Egertova, 2001) and evidence that they may have opposite effects on these receptors (Pertwee, 2008). 

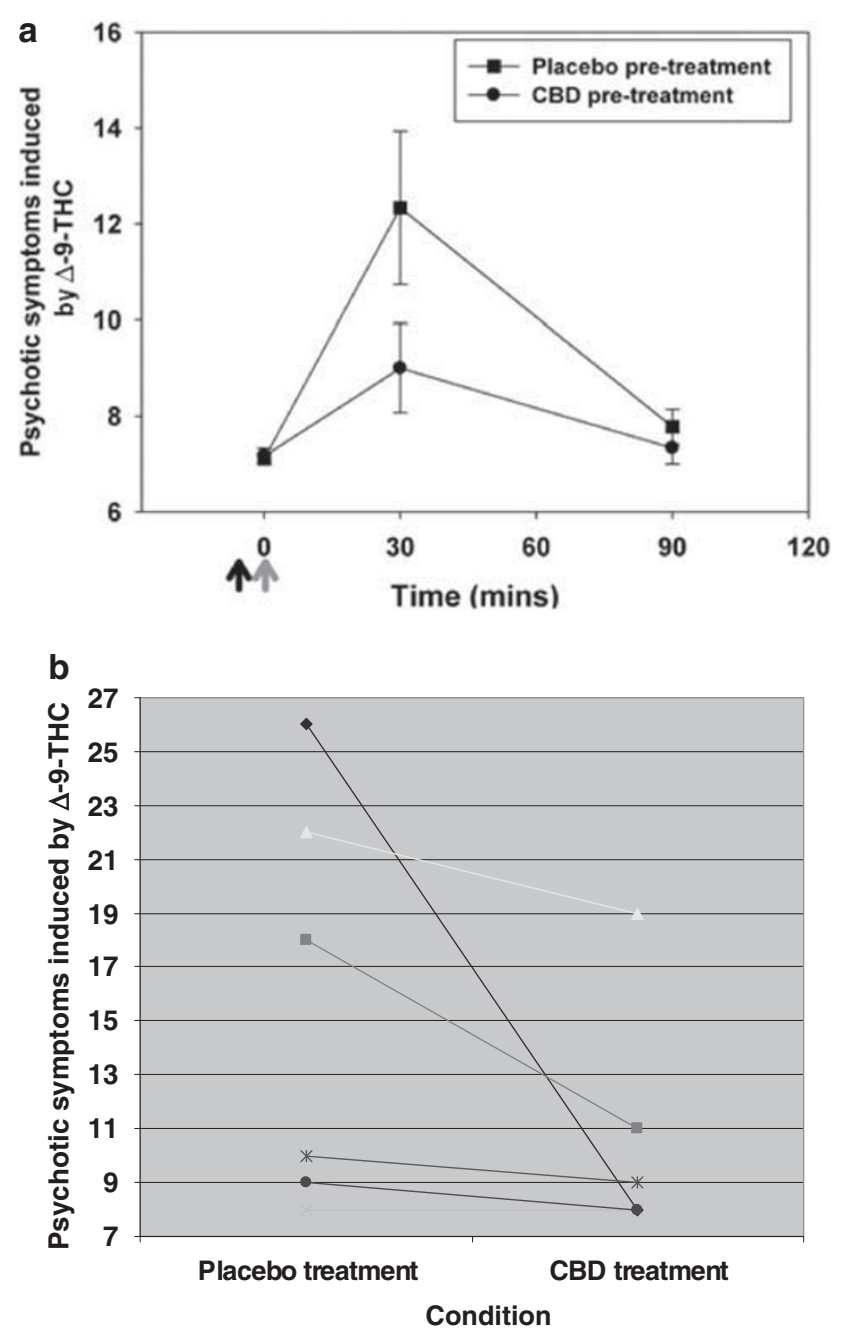

Figure 5 (a) Plots (mean \pm SEM) showing that pretreatment with CBD attenuates the severity of psychotic symptoms (PANSS positive subscale) induced by $\Delta-9-\mathrm{THC}$. Administration of $\Delta-9$-THC, $1.25 \mathrm{mg}$ IV (red arrow) was immediately preceded by administration of either placebo or CBD, $5 \mathrm{mg}$ IV (black arrow). (b) Plots showing PANSS positive subscale ratings for individual subjects $30 \mathrm{~min}$ after administration of $\Delta-9-\mathrm{THC}$ following placebo pretreatment and CBD pretreatment. The corresponding rating at 'time 0 ' (before the administration of $\Delta-9-\mathrm{THC}$ ) for all the subjects under both the pretreatment conditions was 8 (not shown here). The color reproduction of this figure is available on the html full text version of the manuscript.

In certain regions, the differences in the effects of $\Delta-9$ THC and CBD on regional activation were correlated with their differential symptomatic effects. The effect of CBD on amygdalar activation during fear processing was correlated with its reduction of autonomic arousal, as indexed by skin conductance, and there was a trend for a correlation with a reduction of anxiety. $\Delta-9$-THC had the opposite effect on amygdalar activation, and this was correlated with the concurrent increase in the level of anxiety symptoms. The effect of $\Delta$-9-THC on activation in the striatum during verbal recall was directly correlated with its concurrent effect on psychotic symptoms. Although CBD had the opposite effect on striatal activation, it had no effect on psychotic symptoms, and there was thus no analogous correlation between them. This is consistent with evidence that CBD may have antipsychotic effects in patients who already have psychotic symptoms (Zuardi et al, 1982), but not in healthy subjects without pre-exisiting psychotic phenomena.

Modulation of striatal and amygdala activation by $\Delta-9$ THC where its effects correlated with concurrently induced psychotic and anxiety symptoms may underlie the acute induction of psychotic and anxiety symptoms, respectively, by cannabis. Opposite effect of CBD on neural activation in these regions may indicate a potential beneficial effect of CBD in treating these symptoms. This is further supported by the blockade of the emergence of psychotic symptoms by CBD pretreatment in all of the three volunteers in whom $\Delta$-9-THC administration in combination with placebo pretreatment resulted in the induction of psychotic symptoms, during the behavioral experiment using an intravenous route of administration. These results are consistent with the study by Zuardi et al (1982), who showed that combined administration of CBD and $\Delta-9-$ THC resulted in the reduction of paranoia induced by $\Delta-9-\mathrm{THC}$ alone and are also consistent with complementary evidence of potential antipsychotic effects of CBD (Zuardi et al, 2006; Morgan and Curran, 2008). However, this study improves on the study by Zuardi et al (1982) by using standardized rating scales to formally assess the severity of psychotic symptoms. We adopted a pretreatment design, which is effectively similar to the combined administration design used by Zuardi et al (1982), as we believed that it would be more elegant if we could show the emergence of psychotic symptoms with subsequent $\Delta-9$-THC administration. Although there is no reason to believe that CBD would not have a beneficial effect on $\Delta-9$-THC induced psychotic symptoms if administered subsequently, this would need to be tested in a separate study using an appropriate design.

However, the results of these studies need to be considered as preliminary in light of some of the potential limitations. First, the behavioral experiments were conducted in a small sample. Hence, one needs to be cautious about interpreting the results of the behavioral experiment.

As all the cognitive activation tasks performed during the fMRI experiments were presented in the same order, it is also possible that there was some kind of order effect on the patterns of activation during a task. However, although this might have influenced the pattern of activation during a task, it should not have affected the differences between the effects of $\Delta$-9-THC and CBD on activation, as the same order was repeated across the drug conditions. If there was an order effect on activation, it would be the same for both the drugs.

Similarly, it is possible that change in the blood levels of CBD over time during a single scanning session may have affected the patterns of activation during the tasks differentially, such that activation during the tasks performed later in a scanning session might have been modulated to a greater extent by CBD than those performed earlier. However, although this might have influenced the relative effects of CBD on the four cognitive activation tasks, it would not have affected the differences between the effects of $\Delta-9$-THC and CBD on activation during any of the tasks, as all subjects would have been affected in the same manner because the same order of tasks was repeated across all subjects and all drug conditions. 
Lack of symptomatic effects of CBD during the fMRI experiments also makes it difficult to interpret the changes observed at the level of the brain. This may reflect the higher sensitivity of neuroimaging techniques to detect changes at the physiological level in the absence of obvious behavioral changes. It may also indicate that the effects of CBD on symptoms and behavior may be manifested only when it is administered to symptomatic as opposed to asymptomatic/healthy individuals, consistent with earlier human and animal research (Karniol and Carlini, 1973; Zuardi et al, 1982; Zuardi et al, 1993).

It is also possible that the opposite neural effects of these two cannabinoids as measured by the BOLD response in this study may reflect their opposite effects on cerebral blood flow as opposed to opposite effects on neuronal activity. However, the effects of the drugs were specific to both the task conditions as well as the cerebral regions and the analysis additionally controlled for variation in global blood flow. Further, in this study, there was no significant difference in the effects of the cannabinoids on peripheral cardiovascular measures like heart rate and blood pressure, though $\Delta$-9-THC increased heart rate at a trend level of significance relative to placebo (data not shown). A further caveat is that the BOLD response measured using fMRI is a measure of the local vascular response to neuronal activity and opposite effects of the cannabinoids on the BOLD response in several brain regions as observed in this study may not reflect opposite pharmacological effects on the neurons in those brain regions or the neurocognitive processes subserved by them, but may be the net result of their effect on different neuronal populations or neural processes. Hence, this study may provide proof of principle for future studies that examine the effects of CBD in clinical populations (anxiety and psychotic disorders) and pharmacological challenge studies that use complementary neuroimaging techniques like arterial spin labeling and EEG with BOLD fMRI as well as pharmacological blocking studies involving $\triangle-9$-THC and CBD in larger samples.

Overall, these data indicate that some of the effects of CBD on brain function and psychiatric symptoms are in the opposite direction to those of $\Delta-9$-THC. How this might be achieved at a molecular level is uncertain. Although the effects of $\Delta$-9-THC are clearly mediated by central CB1 receptors (Matsuda et al, 1990; Pertwee, 2008), CBD may act through a range of other mechanisms, including inhibition of the uptake and hydrolysis of Anadamide (Mechoulam et al, 2007). However, recent data suggest that CBD can also modulate the effect of $\Delta-9$-THC by binding to CB1 receptors (Pertwee, 2008). Our data are consistent with a potential therapeutic role for $\mathrm{CBD}$ in ameliorating the psychiatric consequences of cannabis use in the general population, and in patients with existing psychiatric disorders (Zuardi, 2008), particularly as conventional antipsychotic medication is relatively ineffective for such conditions (D'Souza et al, 2008). It might also have a role in the treatment of psychotic and anxiety disorders independent of cannabis use (Zuardi, 2008). From a public health point of view, one worrying implication of our results is that cannabis users may be at an increased risk of acute adverse psychological reactions following cannabis use, in light of the increasingly potent forms of cannabis with decreasing CBD content available on the street (Potter et al, 2008).

\section{ACKNOWLEDGEMENTS}

This work was supported by a grant from the Psychiatry Research Trust, UK. Sagnik Bhattacharyya is supported by a Joint MRC/Priory Clinical research training fellowship from the Medical Research Council, UK. Paul Morrison is supported by the Medical Research Council, UK, the Biomedial Research Centre and the Beckley Foundation. Jose A Crippa is the recipient of a Conselho Nacional de Desenvolvimento Científico e Tecnológico (CNPq, Brazil) Productivity fellowship. We thank Glynis Ivin for help with the blinding procedure, storage and dispensing of the drugs. We thank Dr Katya Rubia and Dr Simon Surguladze for allowing us to use the fearful faces and the response inhibition paradigms, respectively.

\section{DISCLOSURE}

The authors report no potential conflicts of interest. Robin M Murray has a financial association (speaking engagements) with the following commercial organizations: AstraZeneca, Bristol-Myers Squibb, Eli Lilly, Janssen (Johnson \& Johnson), Lundbeck, Otsuka, Organon, Pfizer and Servier. Shitij Kapur has had in the past 3 years a financial association with the following commercial organizations: (grant support) AstraZeneca, Bristol-Myers Squibb, GlaxoSmithKline; (consultant/ scientific adviser/speaking engagements) AstraZeneca, Bioline, Bristol-Myers Squibb, Eli Lilly, Janssen (Johnson \& Johnson), Lundbeck, Otsuka, Organon, Pfizer, Servier and Solvay Wyeth.

\section{REFERENCES}

Bhattacharyya S, Fusar-Poli P, Borgwardt S, Martin-Santos R, Nosarti C, O'Carroll C et al (2009). Modulation of mediotemporal and ventrostriatal function in humans by Delta9tetrahydrocannabinol: a neural basis for the effects of Cannabis sativa on learning and psychosis. Arch Gen Psychiatry 66: 442-451. Borgwardt SJ, Allen P, Bhattacharyya S, Fusar-Poli P, Crippa JA, Seal ML et al (2008). Neural basis of Delta-9-tetrahydrocannabinol and cannabidiol: effects during response inhibition. Biol Psychiatry 64: 966-973.

Breiter HC, Etcoff NL, Whalen PJ, Kennedy WA, Rauch SL, Buckner RL et al (1996). Response and habituation of the human amygdala during visual processing of facial expression. Neuron 17: 875-887.

Bullmore E, Long C, Suckling J, Fadili J, Calvert G, Zelaya F et al (2001). Colored noise and computational inference in neurophysiological (fMRI) time series analysis: resampling methods in time and wavelet domains. Hum Brain Mapp 12: 61-78.

Cabeza R, St Jacques P (2007). Functional neuroimaging of autobiographical memory. Trends Cogn Sci 11: 219-227.

Crippa JA, Zuardi AW, Garrido GE, Wichert-Ana L, Guarnieri R, Ferrari L et al (2004). Effects of cannabidiol (CBD) on regional cerebral blood flow. Neuropsychopharmacology 29: 417-426.

Dalton WS, Martz R, Lemberger L, Rodda BE, Forney RB (1976). Influence of cannabidiol on delta-9-tetrahydrocannabinol effects. Clin Pharmacol Ther 19: 300-309.

Doeller CF, King JA, Burgess N (2008). Parallel striatal and hippocampal systems for landmarks and boundaries in spatial memory. Proc Natl Acad Sci USA 105: 5915-5920.

D’Souza DC, Perry E, MacDougall L, Ammerman Y, Cooper T, $\mathrm{Wu}$ YT et al (2004). The psychotomimetic effects of intravenous delta-9-tetrahydrocannabinol in healthy individuals: implications for psychosis. Neuropsychopharmacology 29: 1558-1572. 
D’Souza DC, Abi-Saab WM, Madonick S, Forselius-Bielen K, Doersch A, Braley G et al (2005). Delta-9-tetrahydrocannabinol effects in schizophrenia: implications for cognition, psychosis, and addiction. Biol Psychiatry 57: 594-608.

D’Souza DC, Braley G, Blaise R, Vendetti M, Oliver S, Pittman B et al (2008). Effects of haloperidol on the behavioral, subjective, cognitive, motor, and neuroendocrine effects of Delta-9-tetrahydrocannabinol in humans. Psychopharmacology (Berl) 198: 587-603.

Elphick MR, Egertova M (2001). The neurobiology and evolution of cannabinoid signalling. Philos Trans R Soc Lond B Biol Sci 356: 381-408.

Fadda P, Robinson L, Fratta W, Pertwee RG, Riedel G (2004). Differential effects of THC- or CBD-rich cannabis extracts on working memory in rats. Neuropharmacology 47: 1170-1179.

Fleck MS, Daselaar SM, Dobbins IG, Cabeza R (2006). Role of prefrontal and anterior cingulate regions in decision-making processes shared by memory and nonmemory tasks. Cereb Cortex 16: 1623-1630.

Folstein MF, Luria R (1973). Reliability, validity, and clinical application of the Visual Analogue Mood Scale. Psychol Med 3: 479-486.

Fusar-Poli P, Crippa JA, Bhattacharyya S, Borgwardt SJ, Allen P, Martin-Santos R et al (2009). Distinct effects of $\Delta 9$-tetrahydrocannabinol and cannabidiol on neural acti. Arch Gen Psychiatry 66: 95-105.

Hall W, Solowij N (1998). Adverse effects of cannabis. Lancet 352: 1611-1616.

Hampson AJ, Grimaldi M, Axelrod J, Wink D (1998). Cannabidiol and (-)Delta9-tetrahydrocannabinol are neuroprotective antioxidants. Proc Natl Acad Sci USA 95: 8268-8273.

Haxby JV, Grady CL, Horwitz B, Ungerleider LG, Mishkin M, Carson RE et al (1991). Dissociation of object and spatial visual processing pathways in human extrastriate cortex. Proc Natl Acad Sci USA 88: 1621-1625.

Ilan AB, Gevins A, Coleman M, ElSohly MA, de Wit H (2005). Neurophysiological and subjective profile of marijuana with varying concentrations of cannabinoids. Behav Pharmacol 16: 487-496.

Karniol IG, Carlini EA (1973). Pharmacological interaction between cannabidiol and delta 9-tetrahydrocannabinol. Psychopharmacologia 33: 53-70.

Karniol IG, Shirakawa I, Kasinski N, Pfeferman A, Carlini EA (1974). Cannabidiol interferes with the effects of delta 9-tetrahydrocannabinol in man. Eur J Pharmacol 28: 172-177.

Kay SR, Fiszbein A, Opler LA (1987). The positive and negative syndrome scale (PANSS) for schizophrenia. Schizophr Bull 13: 261-276.

Lastres-Becker I, Molina-Holgado F, Ramos JA, Mechoulam R, Fernandez-Ruiz J (2005). Cannabinoids provide neuroprotection against 6-hydroxydopamine toxicity in vivo and in vitro: relevance to Parkinson's disease. Neurobiol Dis 19: 96-107.

Matsuda LA, Lolait SJ, Brownstein MJ, Young AC, Bonner TI (1990). Structure of a cannabinoid receptor and functional expression of the cloned cDNA. Nature 346: 561-564.

McDonald J, Schleifer L, Richards JB, de Wit H (2003). Effects of THC on behavioral measures of impulsivity in humans. Neuropsychopharmacology 28: 1356-1365.

McLellan AT, Kushner H, Metzger D, Peters R, Smith I, Grissom G et al (1992). The Fifth Edition of the Addiction Severity Index. J Subst Abuse Treat 9: 199-213.

Mechoulam R, Peters M, Murillo-Rodriguez E, Hanus LO (2007). Cannabidiol-recent advances. Chem Biodivers 4: 1678-1692.

Mechoulam R, Spatz M, Shohami E (2002). Endocannabinoids and neuroprotection. Sci STKE 2002: RE5.
Moore TH, Zammit S, Lingford-Hughes A, Barnes TR, Jones PB, Burke $M$ et al (2007). Cannabis use and risk of psychotic or affective mental health outcomes: a systematic review. Lancet 370: $319-328$.

Morgan CJ, Curran HV (2008). Effects of cannabidiol on schizophrenia-like symptoms in people who use cannabis. Br J Psychiatry 192: 306-307.

Morris JS, Frith CD, Perrett DI, Rowland D, Young AW, Calder AJ et al (1996). A differential neural response in the human amygdala to fearful and happy facial expressions. Nature 383: 812-815.

Nelson HE (1982). National Adult Reading Test (NART): Test Manual. NFER-Nelson: Windsor.

O’Leary DS, Block RI, Koeppel JA, Flaum M, Schultz SK, Andreasen NC et al (2002). Effects of smoking marijuana on brain perfusion and cognition. Neuropsychopharmacology 26: 802-816.

Pertwee RG (2008). The diverse CB1 and CB2 receptor pharmacology of three plant cannabinoids: delta9-tetrahydrocannabinol, cannabidiol and delta9-tetrahydrocannabivarin. Br J Pharmacol 153: 199-215.

Phan KL, Angstadt M, Golden J, Onyewuenyi I, Popovska A, de Wit $\mathrm{H}$ (2008). Cannabinoid modulation of amygdala reactivity to social signals of threat in humans. J Neurosci 28: 2313-2319.

Potter DJ, Clark P, Brown MB (2008) Potency of delta 9-THC and other cannabinoids in cannabis in England in 2005: implications for psychoactivity and pharmacology. J Forensic Sci 53: 90-94

Price CJ, Wise RJ, Warburton EA, Moore CJ, Howard D, Patterson $\mathrm{K}$ et al (1996). Hearing and saying. The functional neuroanatomy of auditory word processing. Brain 119(Pt 3): 919-931.

Ramaekers JG, Moeller MR, van Ruitenbeek P, Theunissen EL, Schneider E, Kauert G (2006). Cognition and motor control as a function of Delta9-THC concentration in serum and oral fluid: limits of impairment. Drug Alcohol Depend 85: 114-122.

Rubia K, Russell T, Overmeyer S, Brammer MJ, Bullmore ET, Sharma $\mathrm{T}$ et al (2001). Mapping motor inhibition: conjunctive brain activations across different versions of go/no-go and stop tasks. Neuroimage 13: 250-261.

Sarne Y, Mechoulam R (2005). Cannabinoids: between neuroprotection and neurotoxicity. Curr Drug Targets CNS Neurol Disord 4: $677-684$

Simons JS, Gilbert SJ, Owen AM, Fletcher PC, Burgess PW (2005). Distinct roles for lateral and medial anterior prefrontal cortex in contextual recollection. J Neurophysiol 94: 813-820.

Spielberger C (1983). Manual of the State-Trait Anxiety Inventory. Consulting Psychologists Press Inc: Palo Alto, CA.

Talairach J, Tournoux P (1988). Co-planar Stereotaxic Atlas of the Human Brain. Thieme Medical: New York.

Tart CT (1970). Marijuana intoxication common experiences. Nature 226: 701-704

Viveros MP, Llorente R, Moreno E, Marco EM (2005). Behavioural and neuroendocrine effects of cannabinoids in critical developmental periods. Behav Pharmacol 16: 353-362.

Zuardi AW (2008). Cannabidiol: from an inactive cannabinoid to a drug with wide spectrum of action. Rev Bras Psiquiatr 30: 271-280.

Zuardi AW, Cosme RA, Graeff FG, Guimaraes FS (1993). Effects of ipsapirone and cannabidiol on human experimental anxiety. J Psychopharmacol 7: 82-88.

Zuardi AW, Shirakawa I, Finkelfarb E, Karniol IG (1982). Action of cannabidiol on the anxiety and other effects produced by delta 9-THC in normal subjects. Psychopharmacology (Berl) 76: 245-250

Zuardi AW, Hallak JE, Dursun SM, Morais SL, Sanches RF, Musty RE et al (2006). Cannabidiol monotherapy for treatmentresistant schizophrenia. J Psychopharmacol 20: 683-686. 